\title{
Enhancement of conjugate heat transfer from electronic chips with a rotating tri-vane assembly
}

\author{
R. I. Bourisli \\ Department of Mechanical Engineering, Kuwait University, Kuwait
}

\begin{abstract}
The enhancement of heat transfer from heat-dissipating devices is investigated. The laminar convection-conduction heat transfer from five heat-generating block in a channel at different Reynolds numbers is numerically simulated using the finite volume method. Newly proposed tri-vane structures with constant angular velocities are placed near the upper downstream corners of the blocks. The vanes in the structures drive significant portions of the core channel flow into the dead zones between the blocks. An optimum angular velocity for the structure is shown to exist, giving a reduction in maximum temperature of $16.3 \%$ at $\mathrm{Re}=1000$. The location of the optimum angular velocity was shown to bifurcate because of the influence of the natural frequency of the structure. It is concluded that this effect can have a positive effect on the overall heat transfer from the blocks.
\end{abstract}

Keywords: Heat transfer enhancement, corrugated channel, heated blocks, rotating vanes, optimum angular velocity.

\section{Introduction}

The problem of enhancing heat transfer in channels with geometrical inhomogeneities is of great interest to engineers from both the theoretical and practical points of view. Flow in a channel with heat-dissipating elements, for example, is found in many engineering devices such as compact heat exchangers, oxygenators, dialyzers, nuclear reactor cores, and cooling systems of electronic equipment. In most of these applications the design requires that the maximum temperature of the heat-dissipating components be maintained below a certain temperature in order increase device efficiency and reliability and avoid failure [1]. 
A simple way of accomplishing this objective is to increase the flow rate and thus the rate of heat removal. While this solution is acceptable for low flow-rate devices, the ensuing noise, vibration and pressure drop requirement deems it impractical for many moderate-to-high flow-rate devices.

A common alternative is to modify the channel topology so as to enhance heat transfer from thermally stagnant zones. Among these strategies are altering the configuration of the heated elements [2,3] and adding cylinders and deflectors $[4,5]$ in the channel. In most cases, while moderate heat transfer enhancement is observed relative to the basic configuration, new, thermally-stagnant zones develop in the channel. Additionally, the size and location of the new objects have to be optimized carefully to effect the highest gain in heat transfer for the effort spent.

The effect of adding a streamwise, pulsating component to the flow on the heat transfer has also been proposed by a number of researchers [6-8]. The enhancement in heat transfer was strongly dependent on whether the Strouhal number matched the natural frequency of vortex formation and ejection from inter-block cavities. Enhancements are also directly related to the amplitude of the pulsation, which could not be increased indefinitely. On top of that, the fact that additional power and some sort of variable-frequency pump was required takes away from the practicality of such strategy. Some researchers used oscillating objects, such as plates and cylinder, within the channel to help break the eddies formed between heated blocks $[9,10]$. The same dependence on frequency was observed but with much better heat transfer than when the flow itself is oscillated.

One popular approach among researchers is to use vanes or deflectors to alter the dynamics of the flow around heated blocks [11,12]. Specifically, the vanes lead the cooling fluid into the relatively stagnant zones between the block in order to increase heat transfer from the hidden surfaces, which are normally not greatly affected by the core flow in the channels. While fluid acceleration between the vanes and blocks results in the elimination of large recirculation zones, and thus in heat transfer enhancement, new recirculation zones soon develop, namely between the deflected flow and the upstream surface of the next block in line. The aim of this research is to investigate the utility of adding a rotational velocity component to the vane structure such that the flow is continuously altered. To maintain the effect throughout the rotation period, a tri-vane structure, consisting of three outwardfacing identical vanes, is used.

\section{Problem formulation}

The conjugate conduction/convection problem considered is that of a 2-D, transient, incompressible, Newtonian flow in a channel of height $H$. The fluid flows over five homogeneous $w \times h$ blocks with constant thermal conductivity $k_{s}$, heated from below with a uniform heat flux, simulating electronic chips. The distance from the inlet to the upstream face of the first block is $L_{i}$ and the distance from the downstream face of the last block to the exit is $L_{e}$. The inter-block spacing is $s$. The adiabatic tri-vanes structures are formed by three identical spline curves inscribed in a circle of radius $d$. The center of the circle is located a distance $x$ 


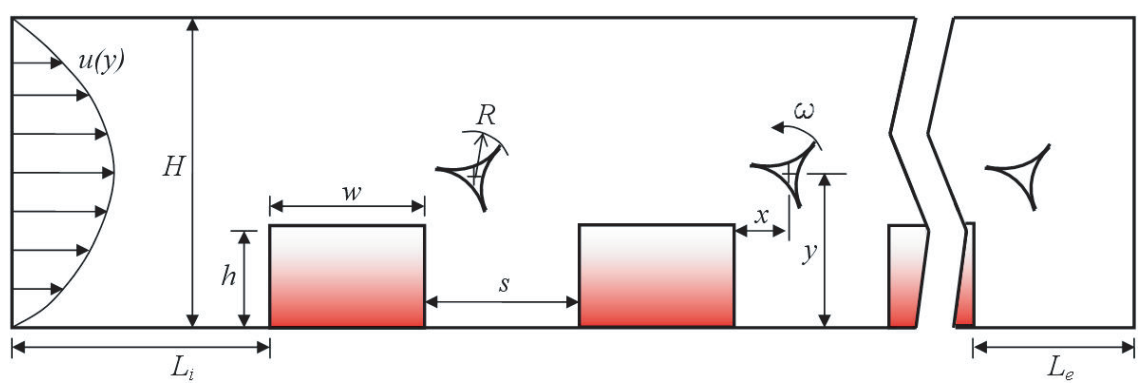

Figure 1: Schematic of the problem showing key dimensional variables.

from the upper left corner of the block, at height $y$ from the bottom surface of the channel. The structure rotates with a constant angular velocity $\omega$. The fluid has density $\rho$, viscosity $\mu$, thermal conductivity $k_{f}$ and Prandtl number Pr. It enters the channel with a parabolic, fully-developed profile, $u_{i}(y)$, and exits with the same profile to zero gage pressure. All properties are assumed constant, and viscous dissipation and body forces are neglected. Figure 1 shows a schematic drawing of the problem showing key geometrical features.

Fluid flow is governed by the 2-D, transient, incompressible Navier-Stokes equations, while energy flow is governed by the transient, 2-D heat equation. These equations can be non-dimensionalized using the following quantities,

$$
\begin{gathered}
u^{*} \equiv \frac{u}{U}, \quad v^{*} \equiv \frac{v}{U}, \quad x^{*} \equiv \frac{x}{H}, \quad y^{*} \equiv \frac{y}{H}, \quad p^{*} \equiv \frac{p H}{\mu U}, \quad t^{*} \equiv \frac{t U}{H} \\
k^{*} \equiv \frac{k_{s}}{k_{f}}, \quad \theta \equiv \frac{T-T_{i n}}{q^{\prime \prime} H / k_{f}}, \quad R e_{H} \equiv \frac{\rho U H}{\mu}, \quad \operatorname{Pr} \equiv \frac{c_{p} \mu}{k_{f}}
\end{gathered}
$$

where $u$ and $v$ are the Cartesian velocity components, $\mu$ is the dynamic viscosity, $c_{p}$ is the specific heat, $k_{f}, k_{s}$, and $k^{*}$ are the fluid, solid and dimensionless thermal conductivities, respectively, and $T_{i n}$ and $\theta$ are the inlet and dimensionless temperatures, respectively. The resulting nondimensional Navier-Stokes equations are,

$$
\begin{aligned}
\frac{\partial u^{*}}{\partial x^{*}}+\frac{\partial v^{*}}{\partial y^{*}} & =0 \\
R e_{H}\left(\frac{\partial u^{*}}{\partial t^{*}}+u^{*} \frac{\partial u^{*}}{\partial x^{*}}+v^{*} \frac{\partial u^{*}}{\partial y^{*}}\right) & =-\frac{\partial p^{*}}{\partial x^{*}}+\frac{\partial^{2} u^{*}}{\partial x^{* 2}}+\frac{\partial^{2} u^{*}}{\partial y^{* 2}} \\
R e_{H}\left(\frac{\partial v^{*}}{\partial t^{*}}+u^{*} \frac{\partial v^{*}}{\partial x^{*}}+v^{*} \frac{\partial v^{*}}{\partial y^{*}}\right) & =-\frac{\partial p^{*}}{\partial y^{*}}+\frac{\partial^{2} v^{*}}{\partial x^{* 2}}+\frac{\partial^{2} v^{*}}{\partial y^{* 2}}
\end{aligned}
$$


and the nondimensional energy equation for the fluid is,

$$
\frac{\partial \theta_{f}}{\partial t^{*}}+u^{*} \frac{\partial \theta_{f}}{\partial x^{*}}+v^{*} \frac{\partial \theta_{f}}{\partial y^{*}}=\frac{1}{R e_{H} \operatorname{Pr}_{f}}\left(\frac{\partial^{2} \theta_{f}}{\partial x^{* 2}}+\frac{\partial^{2} \theta_{f}}{\partial y^{* 2}}\right)
$$

and for the solid blocks,

$$
\frac{\partial \theta_{s}}{\partial t^{*}}+\frac{\partial^{2} \theta_{s}}{\partial x^{* 2}}+\frac{\partial^{2} \theta_{s}}{\partial y^{* 2}}=0
$$

The fluid enters the domain with the fully-developed profile $u_{i}(y)$ and temperature $T_{i}$. At the channel exit, zero streamwise velocity and temperature gradients are imposed. The exposed parts of the lower and upper channel walls are assumed adiabatic with the no-slip boundary condition prescribed. The five blocks experience a constant heat flux of magnitude $q^{\prime \prime} \mathrm{W} / \mathrm{m}^{2}$ through their bottom surfaces. The no-slip boundary condition is prescribed at the left, right and upper surfaces of blocks. Thermally, these interfaces between the blocks and the flow have temperature and heat flux continuities and experience conjugate heat transfer. The boundary conditions can be summarized as follows:

Inlet:

$$
u^{*}=6 y^{*}\left(1-y^{*}\right), \quad v^{*}=\theta_{f}=0
$$

Exit:

$$
\frac{\partial u^{*}}{\partial x^{*}}=\frac{\partial v^{*}}{\partial y^{*}}=\frac{\partial \theta_{f}}{\partial x^{*}}=0
$$

Exposed channel walls:

$$
u^{*}=v^{*}=\frac{\partial \theta_{f}}{\partial y^{*}}=0
$$

Blocks bases:

$$
q^{\prime \prime}=1
$$

Exposed blocks surfaces:

$$
u^{*}=v^{*}=0, \quad \theta_{f}=\theta_{s}, \quad \frac{\partial \theta_{f}}{\partial \mathbf{n}^{*}}=k^{*} \frac{\partial \theta_{s}}{\partial \mathbf{n}^{*}}
$$

Finally, the five tri-vane structures were created and meshed separately and then merged with the channel and block geometry/mesh using the T-Grid software. The five (tri-vane) separate continua were given a constant rotational component of magnitude $\omega$, acting as a temporal boundary condition. A sliding mesh technique was used to ensure continuity of flow between the two main channel and tri-vanes regions. 


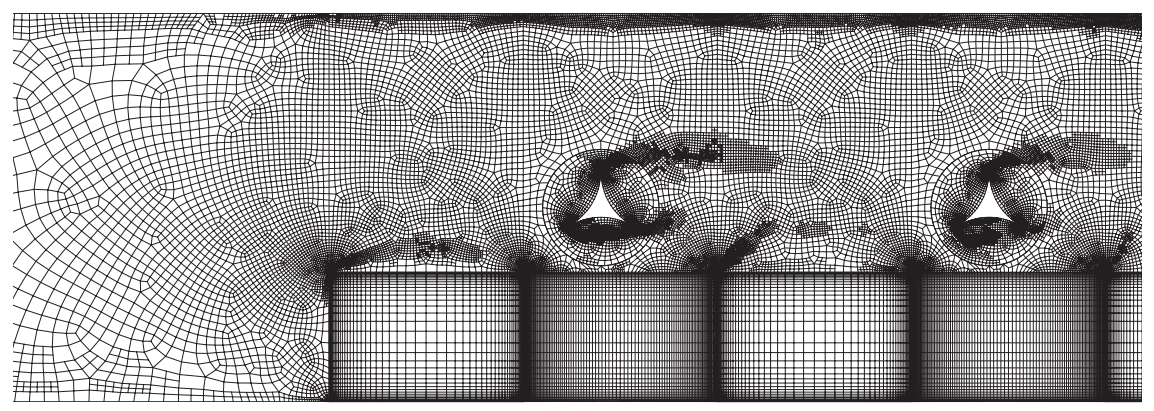

Figure 2: Part of the mesh used showing the effect of adaption.

\subsection{Discretization technique}

The governing equations and boundary conditions were discretized using the finite volume technique [13]. The power law scheme was used to discretize the momentum and energy equations while a first-order upwind scheme was used for the continuity (pressure) equations. A 1st-order, implicit formulation was used for the transient term. The problem of flow over five heated blocks without vanes was examined using a series of meshes, ranging from 80,000 to 197,900 cells. The maximum dimensionless temperature and the area-weighted average Nusselt number values for the first and fifth blocks were used to assess the quality of the mesh. For meshes larger than 170,000 cells, changes in all three quantities cease to be greater than $1 \%$. In most results reported here, 180,500 cells were used. A series of mesh adaption modifications were performed at different intervals within a rotation period to ensure that the mesh is optimized with respect to high velocity and temperature gradients. A partial view of a sample resulting mesh, showing the effect of adaption, is shown in Figure 2. The consistency of the numerical algorithm was tested by comparing its results for a single block to the "steady" ones of Young and Vafai [2]; excellent agreement between the two methods/grids was observed, judging by the maximum temperature and local Nusselt number distributions reported.

\section{Results and discussion}

Simulations were performed at Reynolds numbers 250, 500 and 1000 for a fluid with Prandtl number 0.72 . The heat flux was taken to be $q^{\prime \prime}=1 \mathrm{~W} / \mathrm{m}^{2}$. In all, nine rotational speeds for the tri-vanes were tested: $\omega=0.63,1.05,1.26$, $1.57,2.09,3.14,4.19,5.03$ and $6.28 \mathrm{rad} / \mathrm{s}$, corresponding to rotation periods of $10,6,5,4,3,2,1.5$, and 1.251 , seconds, respectively. The tri-vane geometry, nondimensionlized by $H$, has $d=0.2, x=0.2$ and $y=0.5$. The equations of motion were discretized and solved as aforementioned. Due to the coupled nature of the problem, a coupling of the pressure, momentum and energy equations was necessary at each time step of each of the 27 cases tested. This resulted in 

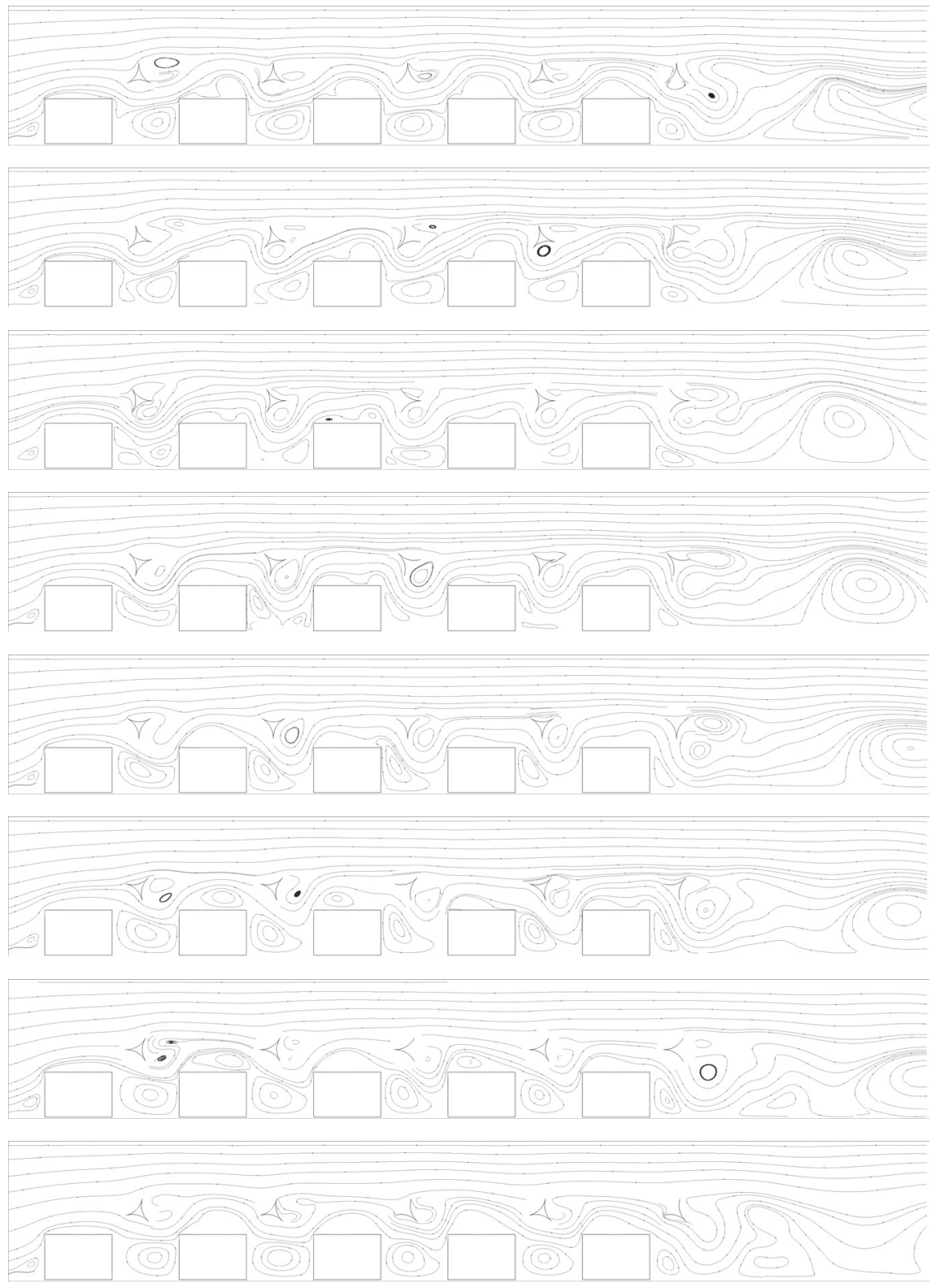

Figure 3: Streamlines through one third of a period, $\mathrm{Re}=1000$. 

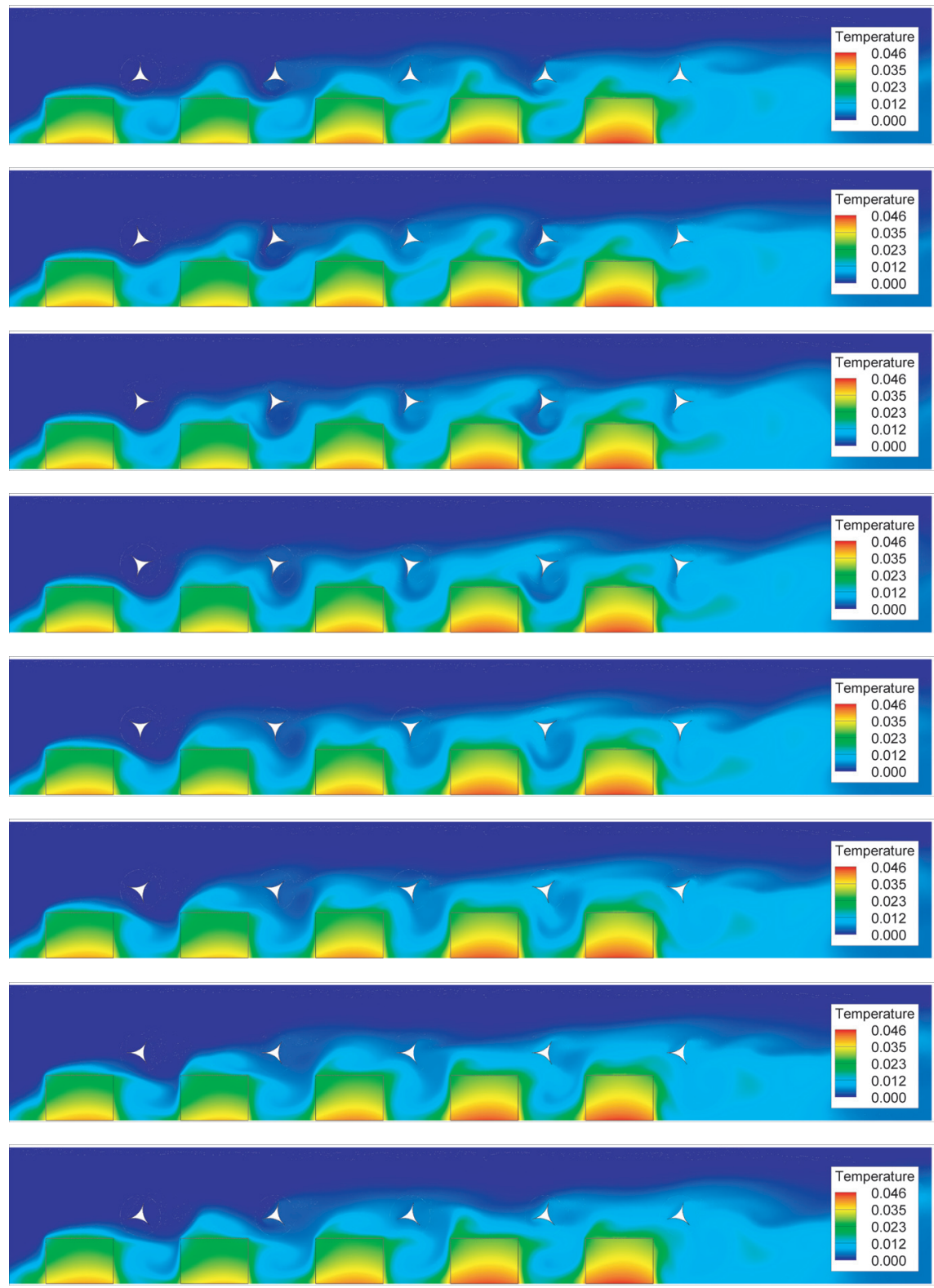

Figure 4: Temperature contours through one third of a period, $\operatorname{Re}=1000$. 


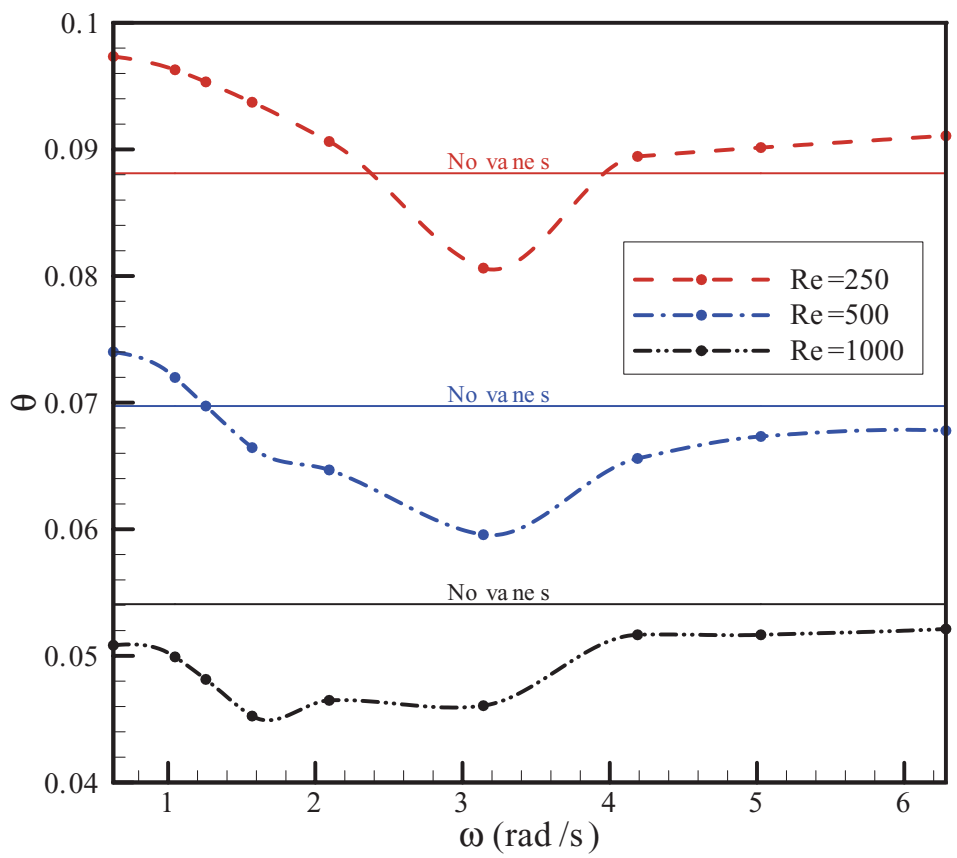

Figure 5: Maximum temperature, $\theta_{\max }$, among the blocks vs. angular velocity, $\omega$.

extremely long (computational) simulation times before cyclic steady state was reached, even though the physical flow became cyclically steady after a relatively short amount of (physical) time.

Figures 3 show the evolution of the streamlines in the channel through one third of a period. Due to the circumferential symmetry of the structure, one third of a complete period is sufficient to draw conclusions. The dynamic nature of the flow is evident through the intermittent flow of fluid from the core into the stagnant regions between the blocks. Large eddies do not form in the grooves and reduce the Nusselt number over the sides of the blocks. Additionally, and more importantly, when the vane facing the block rotates away, an eddy is given a chance to form momentarily before it is swept away by the next vane. This prevents stagnant zones in the whole channel and results in the fluid passing over the next upper surface with even higher momentum.

Figures 4 show the concurrent temperature contours in the channel. The effect of eddy formation and ejection is clearly visible in the areas between blocks, where cold fluid, almost at the temperature of the core flow, penetrates deep into the grooves, carrying with it a large portion of the heat. The penetration of the cool fluid to the bottom of the grooves is of paramount importance because of their proximity to the heat source.

The effect of the angular velocity, $\omega$, on the maximum temperature in the five blocks, $\theta_{\max }$, is shown in figure 5 at the three Reynolds numbers considered. As 
expected, the effectiveness of the tri-vanes, rotating or not, does not enhance heat transfer at low Reynolds numbers. This can be attributed to the fact that at such Reynolds numbers, the flow lacks the required momentum to drive itself into the grooves as the vanes intended. At the same time, the existence of the structure over any block hinders the core flow from attaining its usual core velocity over the top surface of the next block in line, thereby reducing heat transfer. At $R e=250$, only one rotational velocity was successful in reducing the maximum temperature compared to the basic grooved channel. It is expected, but not verified, that this optimum will quickly disappear as the Reynolds number is reduced further. For moderate-to-high (laminar) Reynolds numbers, the use of the tri-vane structures clearly reduces the maximum temperature in the heat-generating blocks. In fact, for $\mathrm{Re}=1000$, any value of $\omega$ results in a reduction in the maximum temperature for the shape studied. In general, reductions in the maximum temperature amongst the blocks were $8.50 \%, 14.6 \%$ and $16.3 \%$, for $\operatorname{Re}=250,500$, and 1000 , respectively.

An important observation can be made regarding the value of the optimum $\omega$ as $\mathrm{Re}$ increases. Interestingly, the clear optimum observed for the $\mathrm{Re}=500$ case gradually bifurcates into two co-optima as the Reynolds number is increased. It is known that in static mode, heat transfer from obstacles placed in a channel is maximized when the frequency of oscillation/rotation is synchronous with the natural frequency of vortex shedding, a phenomenon known as lock-on [14]. It is also reasonable to assume that heat transfer is not maximized over all surfaces simultaneously. We observe here that the resultant effect of the tri-vanes is to force the large vortices to take a different route than the usual one. Thus, the bifurcation can be explained by the fact that the periodicity of vortex shedding is severely disrupted by the rotation of the tri-vanes in such a way that it artificially overrides the natural lock-on and forces the flow into dead zones, normally unattended by the undisturbed flow. In other words, the asynchronous rotation (with respect to the natural frequency) can become so pronounced as to "unexpectedly" enhance heat transfer from different block surfaces, thereby doing more good than harm.

\section{Conclusions and future work}

In this research, enhancement of the laminar convection-conduction heat transfer from five heat-generating blocks in a channel is investigated numerically using the finite volume method. A rotating tri-vane structure was used to drive the flow into the grooves between the blocks. The result was a noticeable decrease in the maximum temperature in the blocks. The periodic diversion of the core channel flow into the grooves prevents sustaining large, stagnant eddies. Reductions in the maximum temperature in the blocks were as high as $16.3 \%$ for $\operatorname{Re}=1000$. An optimum angular velocity for the tri-vanes was shown to exist. It is believed that the rotational velocity "throws off" the natural frequency of vortex shedding. This dilatation of the lock-on effect was shown to have a positive effect on the overall heat transfer from the blocks. For future work in this area, more laminar cases can be simulated to draw more physical conclusions. It is also interesting to see if 
effects of the tri-vanes is as pronounced in the turbulent regime. The tri-vanes, or similar shapes, that move with variables velocities should also be investigated.

\section{References}

[1] Teo, J., Thermal cycling aging effect on the reliability and morphological evolution of $\mathrm{SnAgCu}$ solder joints. IEEE Transactions on Components and Packaging Technologies, 30, pp. 279-284, 2007.

[2] Young, T.J. \& Vafai, K., Convection flow and heat transfer in a channel containing multiple heated obstacles. International Journal of Heat and Mass Transfer, 41, pp. 3279-3298, 1998.

[3] da Silva, A.K., Lorenzini, G. \& Bejan, A., Distribution of heat sources in vertical open channels with natural convection. International Journal of Heat and Mass Transfer, 48(8), pp. 1462-1469, 2005.

[4] Herman, C. \& Kang, E., Comparative evaluation of three heat transfer enhancement strategies in a grooved channel. Heat and Mass Transfer, 37, pp. 563-575, 2001.

[5] Korichi, A., Oufer, L. \& Polidori, G., Heat transfer enhancement in self-sustained oscillatory flow in a grooved channel with oblique plates. International Journal of Heat and Mass Transfer, 52, p. 11381148, 2009.

[6] Moon, J.W., Kim, S.Y. \& Cho, H.H., Frequency-depedent heat transfer enhancement from rectangular heated block array in a pulsating channel flow. International Journal of Heat and Mass Transfer, 48, pp. 4904-4913, 2005.

[7] Korichi, A. \& Oufer, L., Heat transfer enhancement in oscillatory flow in channel with periodically upper and lower walls mounted obstacles. International Journal of Heat and Fluid Flow, 28, p. 10031012, 2007.

[8] Alawadhi, E.M. \& Bourisli, R.I., The role of periodic vortex shedding in heat transfer enhancement for transient pulsatile flow inside wavy channels. Int Journal of Natural Sciences and Engineering, 1(2), pp. 79-85, 2009.

[9] Fu, W.S. \& Tong, B.H., Numerical investigation of heat transfer characteristics of the heated blocks in the channel with a transversely oscillating cylinder. International Journal of Heat and Mass Transfer, 47, pp. 341-351, 2004.

[10] Florio, L.A. \& Harnoy, A., Use of a vibrating plate to enhance natural convection cooling of a discrete heat source in a vertical channel. Applied Thermal Engineering, 27, pp. 2276-2293, 2007.

[11] McGarry, M., Campo, A. \& Hill, D., Numerical simulations of heat and fluid flow in grooved channels with curved vanes. Numerische Heat Transfer, Part A, 46, pp. 41-54, 2004.

[12] Luviano-Ortiz, L., Hernandez-Guerrero, A., Rubio-Arana, C. \& RomeroMendez, R., Heat transfer enhancement in a horizontal channel by the addition of curved deflectors. International Journal of Heat and Mass Transfer, 51, pp. 3972-3984, 2008. 
[13] Patankar, S.V., Numerical Heat Transfer and Fluid Flow. Series in Computational Methods in Mechanics and Thermal Sciences, McGraw Hill: New York, 1980.

[14] Mahir, N. \& Altaç, Z., Numerical investigation of convective heat transfer in unsteady flow past two cylinders in tandem arrangements. International Journal of Heat and Fluid Flow, 29, pp. 1309-1318, 2008. 